\title{
HISTORIA
}

\section{TRAS LAS HUELLAS DE NUESTROS ORÍGENES CUIDADORES}

\author{
Mercedes Núñez del Castillo* \\ Dr. José Siles González**
}

*Enfermera, Antropóloga, Profesora del Departamento de Enfermería de la Universidad de Alicante.

**Catedrático del Departamento de Enfermería de la Universidad de Alicante.

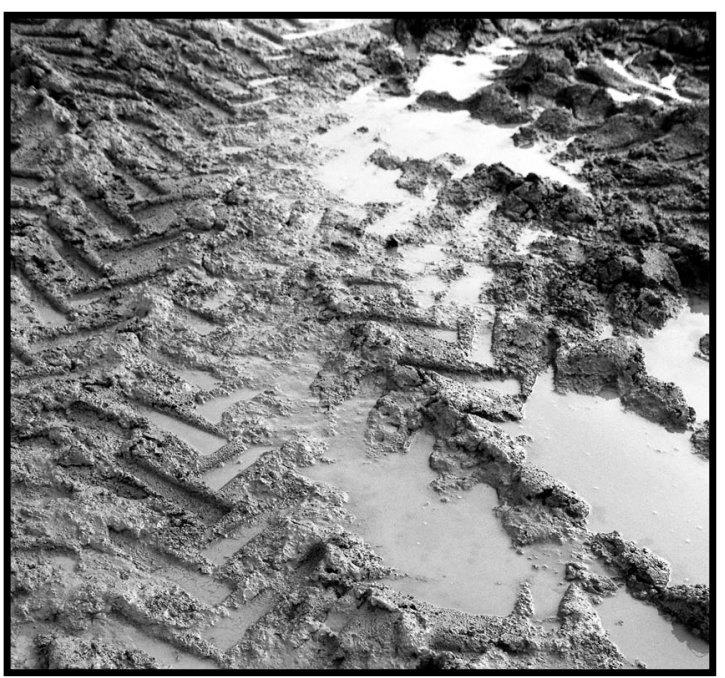

\section{AFTER THE PRINTS OF OUR CARING ORIGINS}

\section{ABSTRACT}

The aim of this work is to demonstrate that iconography becomes a source for nursing history. A descriptive, retrospective, comparative, etnographic historical study was carried out.

The iconographic material used corresponds to cave paintings from the Valencian County, declared world's heritage.

In order to potentiate the hermeneutical process from a nursing perspective, the Henderson's needs system categorisation has been used.
The main conclusion of this study is the demonstration of the pertinence of iconography when it comes to developing historical knowledge of nursing.

Key words: Nursing history, nursing care, anthropology of care, iconography, Henderson needs.

\section{RESUMEN}

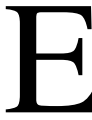
1 objetivo de este estudio, es demostrar que la iconografía constituye una fuente para la historia de la enfermería. Se ha pretendido alcanzar este objetivo realizando un estudio descriptivo, retrospectivo, comparativo, histórico etnográfico.

El material iconográfico utilizado corresponde a las pinturas rupestres que se encuentran en los abrigos y cuevas de la Comunidad Valenciana, declarados patrimonio de la humanidad.

Para potenciar el proceso hermenéutico desde la perspectiva de enfermería se ha empleado la categorización del sistema de necesidades de Henderson.

La conclusión principal de este estudio, es demostrar la pertinencia de la iconografía para desarrollar el conocimiento histórico de la enfermería.

Palabras clave: Historia de la enfermería, cuidados enfermeros, antropología de los cuidados, iconografía, necesidades de Henderson. 


\section{INTRODUCCIÓN}

Se trata de un estudio descriptivo, retrospectivo histórico-etnográfico de los cuidados de enfermería, analizado desde la perspectiva de las necesidades básicas de Virginia Henderson, utilizando como fuente la iconografía.

Alberdi (1983) define la enfermería como:"... una disciplina tan antigua como el propio hombre, dado que tiene que ver con todos aquellos elementos y factores materiales y humanos que convergen en una actividad innata y esencial en el ser humano. Y Siles (1999), la define como el cuidado del individuo, el grupo y la comunidad...".

Henderson, en su definición sobre Enfermería hace hincapié en el cuidado, tanto de los individuos enfermos como sanos, al paciente con actividades esenciales para mantener la salud, recuperarse de la enfermedad o lograr una muerte tranquila.

La independencia del paciente es un criterio importante para la salud, hasta el punto que en sus escritos equipara salud con independencia, e identifica 14 necesidades básicas que son los componentes del cuidado enfermero.

También Collier (1997), estudiosa de la materia dice que "los cuidados son un conjunto de actos de vida, habituales y cotidianos, que tienen por objeto permitir que esta continúe y se desarrolle".

En las comunidades prehistóricas las responsabilidades femeninas estaban relacionadas con la fecundidad, Hernández (1999), y Siles (1998), afirman que las principales responsabilidades femeninas eran satisfacer los recursos cruciales como el mantenimiento de la salud, atención a los partos y cuidado de la prole, siendo todos ellos cuidados de supervivencia, "... la mujer cuidadora se va a ocupar de los cuidados desde la cotidianeidad.." Siles (1998). Asegurando través de la conservación y el aprendizaje la continuidad de la especie y de la vida. De este modo, Hernández (1999), “ “.. el saber empírico se va configurando mediante la praxis de las "tareas asignadas", se establece "el valor social" de los cuidados de supervivencia proporcionados por las mujeres en las primeras comunidades prehistóricas...”, pero además “... los ritos pueden ser considerados antecedentes de los actuales procedimientos en cuidados en salud, estructurando actos ceremoniales" como se puede interpretar en el conjunto iconográfico.
El área de estudio se centra en la Comunidad Valenciana, ya que ha sido fundamental en nuestra cultura y en nuestra historia, el arte rupestre levantino es una manifestación única que corresponde a una sociedad prehistórica que habitó las principales sierras y paisajes montañosos del arco Mediterráneo de la Península Ibérica, es un arte al aire libre, ubicado en abrigos rocosos y acantilados, se da en un paisaje escasamente alterado donde se complementa la creación de la naturaleza y la creación del hombre, de esta forma se reflejan los modos tradicionales de utilización de este territorio, evidenciándose las relaciones sociales desde hace milenios y que se mantienen casi un millón de kilómetros de longitud.

El arte macroesquemático, contestano o lineal figurativo, se descubrió en Pla de Petracos, de cronología prelevantina, neolítico antiguo, y se relaciona con las primeras comunidades agrícolas y ganaderas de la comunidad, las figuras suelen ser de gran tamaño y generalmente gruesos los elementos que lo forman. El tema central, es la figura humana, con los brazos elevados, en posición de orante, que adquiere carácter de ídolo, parece ser exclusivo de la provincia de Alicante.

$\mathrm{El}$ arte macroesquemático es la prueba más evidente de la nueva religiosidad neolítica ligada a preocupaciones de tipo agrícola, adquiriendo los yacimientos el carácter de santuarios.

El arte Levantino, que según Fortea y Jordá, se extiende desde neolítico hasta edad de los metales, se caracteriza por el naturalismo de sus escenas, en las que predomina la caza, la recolección y las de carácter religioso, como danzas rituales u ofrendas, se ubica sobre todo en abrigos de escasa profundidad y paredes rocosas protegidas por una pequeña cornisa, en la parte media y alta de los barrancos. La escena aparece como elemento esencial y los objetos se sitúan ocupando un lugar previamente determinado por el artista en relación con otros objetos que determinan la escena.

\section{Objetivo general}

Demostrar que el material iconográfico constituye una fuente para la historia de la enfermería.

\section{Objetivos específicos.}

Describir y analizar los cuidados de enfer- 
mería desde la perspectiva de V. Henderson en el arte rupestre de la Comunidad Valenciana.

Demostrar que los conocimientos empíricos e intuitivos adquiridos por las cuidadoras de forma oral, se transmiten a través de la iconografía.

Demostrar mediante análisis iconográfico, que la iconografía refleja de forma holística las realidades de los cuidados de la salud.

\section{Hipótesis nuclear}

La iconografía constituye una fuente de estudios para la historia de la enfermería.

\section{Otros puntos de partida}

La iconografía constituye una fuente para analizar los cuidados de enfermería desde la perspectiva de Virginia Henderson

Los conocimientos empíricos e intuitivos adquiridos por las cuidadoras de forma oral están presentes en la iconografía.

La iconografía refleja de forma holística las realidades de los cuidados de la salud.

\section{METODOLOGÍA}

El material iconográfico que se va a utilizar para verificar o refutar las hipótesis son las pinturas rupestres que se encuentran en los 300 abrigos y cuevas de la Comunidad Valenciana, declaradas Patrimonio de la Humanidad, en la categoría de paisajes culturales, el 2 de diciembre de 1998, en el plenario de la UNESCO, celebrado en Kioto (Japón).

La muestra analizada se ha obtenido mediante búsqueda iconográfica, analizando las pinturas de arte levantino y macroesquemático.

Las variables, están distribuidas por categorías y corresponden a las 14 necesidades básicas de Henderson.

\section{Análisis de los datos}

Los datos cualitativos se han recogido a través de la observación de las iconografías seleccionadas, y para analizar las iconografías, se llevan a cabo dos tipos de análisis, el iconográfico propiamente dicho y el antropológico.

Para las iconografías, se va a utilizar la interpretación iconográfica propuesta por Panofsky, (1995).
Para realizar los análisis iconográficos, se va a utilizar los esquemas propuestos por Siles (1999).

\section{RESULTADOS}

Todas las necesidades observadas son de independencia, y siendo la de actuar según sus creencias y valores, comer y beber, mantener una buena postura y la de aprendizaje las más representadas.

La caza y la recolección de miel en LA ARAÑA, el vareado de los almendros en LA SARGA o el amamantamiento en SORELLETS, son actividades que se realizan para satisfacer la necesidad de BEBER Y COMER.

En la escena de amamantamiento de SORELLETS, se observan manifestaciones y actividades relacionadas con la inmediatez del mismo, también por los brazos en jarra y los pechos al descubierto de la figura femenina de gran tamaño. La caza es una actividad física, que influye como factor biológico en esta misma necesidad. De forma indirecta el clima, las creencias o su propia cultura son factores sociológicos presentes y tenidos en cuenta en la independencia de esta necesidad, estando el clima, representado por el sol en uno de los abrigos, teniendo éste además otras connotaciones religiosas.

En la necesidad de MOVERSE Y MANTENER UNA BUENA POSTURA, como factores sociológicos observamos que las figuras en posición de caza, o la del orador en TOURRADES, pueden estar marcadas por costumbres adquiridas. Se observan manifestaciones de independencia representadas por la cabeza recta, sin flexionar, pies en ángulo recto con las piernas, caderas y piernas derechas. En SORELLETS estas posturas podrían estar relacionadas con las plegarias.

En cuanto a las figuras en movimiento, se ven movimientos de baile, ejecución, como estirarse, coger objetos o caminar o correr siendo todas ellas manifestaciones de independencia.

Dentro de las manifestaciones de independencia propuestas por Henderson en la necesidad de VESTIRSE, tendríamos en cuenta la calidad de los vestidos relacionados con el clima, y el significado de los mismos como apariencia o pertenencia, observándose faldas y posiblemente pantalones ajustados y pantalones bombachos.

La representación de los pantalones bombachos ajustados, se puede entender como un factor bioló- 
gico ya que el cazador lleva ropa diferente al orante.

Los adornos que portan, pueden ser considerados como manifestaciones de independencia, aunque también podría ser determinante el status social, en el que llevarían objetos diferenciadores.

Se observan manifestaciones de independencia en la necesidad de EVITAR LOS PELIGROS en TOURRADES, en forma de seguridad física, manifestada por una cerca o una trampa, en la figura que porta el arco y las flechas o las escenas de caza, en LA SARGA y REMIGIA, dado que conoce el entorno donde se mueve y como consecuencia toma medidas para su protección. O como seguridad psicológica, la figura del orante

A través de la evolución, el hombre ha desarrollado diferentes símbolos para expresarse, siendo muy importantes los relacionados con la comunicación no verbal o sea con el leguaje del cuerpo, constituyendo una forma arcaica de transmisión de nuestro significado interior, en PLA DE PETRACOS se observa por la posición, movimientos corporales o por los gestos, enviando un mensaje de su forma de estar o de sus intenciones, siendo la posición de las manos de las figuras factores biológicos, además el conjunto de las figuras también podrían expresar emociones o deseo o incluso una búsqueda de atención, constituyendo estos los factores psicológicos de la manifestación de independencia de la necesidad de comunicación,

La danza en si, las cintas y brazaletes que llevan las mujeres, en el ABRIGO DE LUCIO, los abalorios, o incluso los estuches fálicos de LA SARGA como signo de poder o fertilidad, o la posición orante de los antropomorfos TOURRADES son manifestaciones de independencia de la necesidad ACTUAR SEGÚN SUS CREENCIAS. Los gestos y las actitudes corporales de las mujeres danzantes son considerados como factor biológico o como manifestaciones de independencia.

Se observan acciones como en Cueva REMIGIA la actividad del baile, pues parecen que son danzas rituales o incluso ofrecer o presentar a los niños a la "dea".

Observamos la necesidad de OCUPARSE PARA REALIZARSE sólo mediante actividades como son las de la vida cotidiana, caza y agricultura.

La danza en si, los abalorios, las cintas y bra- zaletes que llevan las mujeres son manifestaciones de la necesidad de RECREACIÓN

Para desarrollar la personalidad de los niños, se realiza el juego del baile, actuando como factor psicológico, mientras que los factores sociológicos que van a actuar son la cultura, los roles sociales o incluso la organización social.

En la necesidad de APRENDIZAJE, la posición del orante, del ABRIC TOURRADES, o la escena del amamantamiento de SORELLETS, determinan un punto de referencia para las manifestaciones de independencia, además el gesto es considerado como un factor biológico que va a influir para satisfacer esta necesidad, también las escenas de caza o recolectoras pueden ser tenidas en cuenta en el proceso de aprendizaje.

\section{DISCUSIÓN}

En la Prehistoria todas las necesidades observadas son en forma de manifestación de independencia, siendo el aprendizaje, la actuación según sus creencias y valores y la necesidad de comer y beber, las más representadas; estas necesidades se manifiestan mediante valores indirectos o instrumentos, siendo los factores biológicos los que van a influir en la satisfacción de las necesidades relacionadas con roles sociales.

La representación de los carpidos haría pensar que es la base de su alimentación, pero MartínCano, (2001) afirma que en la prehistoria los productos de la caza no eran cruciales, lo que pone de manifiesto que la mayoría de los alimentos provenían de la recolección, tarea femenina.

El hombre adulto tiene conocimiento de su entorno y es consciente del peligro, y por tanto toma medidas, siendo este un factor biológico que va a influir en la satisfacción de la necesidad de evitar los peligros, curiosamente, esta necesidad aparece poco representada.

A través de la evolución, el hombre ha desarrollado diferentes símbolos para expresarse, siendo muy importantes los relacionados con la comunicación no verbal o sea con el leguaje del cuerpo, constituyendo una forma arcaica de transmisión de nuestro significado interior.

El aprendizaje como valor sociológico, no es precisamente una necesidad ampliamente observada de forma objetiva, pero sí de forma implícita. 
No se conoce el significado de cada uno de los elementos representados, pero afirma Harris (1999), que parece probable que las cuevas y abrigos sirvieran entre otras funciones para intensificar su sentido de identidad o para educar.

La iconografía se analiza dentro del contexto, ya que el arte rupestre se localiza en su mayor parte en cuevas y abrigos de difícil acceso, lo que según Siles, (1998), avala la teoría mágica de esta actividad.

\section{CONCLUSIONES}

Que la iconografía representa una fuente para estudiar los cuidados de enfermería y la historia de la profesión.

Que la profesión enfermera, en sus diferentes denominaciones como cuidadora intuitiva dedicada a los cuidados de supervivencia ha estado presente en las iconografías analizadas.

Que el origen de los cuidados debemos buscarlo en las actividades de la alimentación.

Que tanto el clima como la religión/creencias y la cultura son factores fundamentales y determinantes en esta etapa.

La iconografía es una fuente para analizar los cuidados desde la perspectiva de Virginia Henderson, siendo las pinturas rupestres las primeras manifestaciones donde se observan estas necesidades.

Que los conocimientos empíricos e intuitivos que han adquirido las cuidadoras de forma oral están presentas en las iconografías.

A pesar que la necesidad de aprendizaje no está muy presente en las iconografías analizadas, éstas suponen un medio directo de aprendizaje.

\section{BIBLIOGRAFÍA}

Abrigo de Albocasser.

http://club.telepolis.com/meugenia1/s_pau_albocasser.htm

ALBERDI, R.M ${ }^{\mathrm{a}}$. et al. (1983): Conceptos de enfermería. UNED, Madrid.

Arte Levantino. www.arterupestre.net

ANATI, E. (2001): Les premiers arts sur la terre. Capítulo de libro : De l'apparition de la vie à l'homme moderne Volumen I. Directores Yves Coppens y Pascal Picq. Anati, E; Barriel, E; Berthelet,A; DeBonis,L; Brunet, M; Chavaillon, J; Hublin,J.J.; Jaeger,J.J.; Picq,P; Senut,B; Thomas, H; Vandermeersch,B. Librairie Arthème Fayard.
BARANDIARAN,I. (1996): El arte prehistórico (139) en: Historia del Arte I. El mundo antiguo. Dirigida por Ramírez J:A. J. Alcina Franch, I Barandiaran, M. Bendala Galan, M.A. Elvira, C. García-Ormaechea, J. Jimenez, R. Olmos, J. Padró. Alianza Editorial S.A. Madrid

BÁRCENA, R. (2001): Arte rupestre del Levante español, monografía realizada en el marco del seminario de "sociedades Cazadoras-Recolectoras del Viejo Mundo", dictado por el autor en el transcurso del primer trimestre del año 2001. html

http://www.monografias.com/trabajos $11 \mathrm{mng} / \mathrm{mng} . \mathrm{s}$

COLLIÈRE, M. (1989). Utilización de la antropología para abordar las situaciones de cuidados. Rol, 179180,17-25.

(1997): Promover la vida. De la práctica de las mujeres cuidadoras a los cuidados de enfermería. Editorial Mc. Graw-Hill Interamericana España.

DE MIGUEL, M ${ }^{\mathrm{a}}$ P. (2004): Aproximación a las manifestaciones funerarias durante la edad del bronce en tierras alicantinas, a través de los restos humanos. Separata; La edad del Bronce, en tierras valencianas y zonas limítrofes, eds: Laura Hernández Alcaraz; Mauro S. Hernández Pérez. ISBN: 84-7784-440-2

CHUECA GOTILLA, F.(1987): Prólogo. Gran historia universal volumen I Prehistoria. Cano Herrea, M; Chapa Brunet, T; Delibes de Catro,G; Moure Romanillo,JA; Querol,MA; Santonja Gomez,M. Editorial Najera, Madrid .

El arte rupestre del arco Mediterráneo de la Península Ibérica. http://www.carm.es/ctyc/patrimoniohistórico/arterupestre/unesco

HARRIS, M. (1999): Introducción a la antropología general. Alianza Editorial. Madrid.

HENDERSON, V. A. (1994): La naturaleza de la enfermería. Una definición y sus repercusiones en la práctica, la investigación y la educación. Reflexiones 25 años después.. Mc Graw-Hill Interamericana de España S.A.

HERNÁNDEZ PÉREZ, M S; (et al). (1988): Arte rupestre en Alicante. Fundación Banco exterior, Banco de Alicante grupo Banco Exterior.

HERNÁNDEZ CONESA, J. (1999): Historia de la Enfermería. Un análisis histórico de los cuidados de Enfermería. Mc. Graw-Hill Interamericana.

JOCHIM, MICHAEL.(1983): Paleolithic Cave Art in Ecological Perspective, en Hunter-Gatherers Economy in Prehistory: A european Perspective, G. Bailey (ed) pp 212 -219. Nueva York, Cambridge University Press

HENDERSON, V. A. (1994): La naturaleza de la enfermería. Una definición y sus repercusiones en la práctica, la investigación y la educación. Reflexiones 25 
años después.. Mc Graw-Hill Interamericana de España S.A.

LAIN ENTRALGO, P. (1978). Historia de la medicina. Editorial Masson, Barcelona.

MARTÍNEZ, R. (2003): Apuntes sobre las pinturas rupestres del parque cultural de Bicorp.www.arterupestre.net

MARTÍN-CANO ABREU, F.B (2001) Falsas ideas sobre los papeles sexuales en la prehistoria, periódico electrónico SEIAAL,

http://colciencias.gov.co/seiaal/documentos/fmca.htm

http://es.geocities.com/culturaarcaica/papeles.sexual es.html

(2001) Recolectoras, danzantes, cazadoras pintadas en escenas artísticas prehistóricas levantinas. Odisea, Rumbo al pasado

http://www.odiseo.es.vg/

MARTÍNEZ, R. (2003): Apuntes sobre las pinturas rupestres del parque cultural de Bicorp.www.arterupestre.net.

MELLARS, PAUL (1985). The ecological Basis of Social Complexity in the Upper Paleolithic of Southwestern France, en Prehistoric Hunter-Gatherers:
The emergence of Cultural Complexity, D.Price J. Brown (eds) pp271 -297.Nueva York, Academic Press.

MOURE ROMANILLO, J. A. (1987): Al arte paleolítico. Capítulo de libro: Gran historia universal volumen I Prehistoria. Cano Herrea, M; Chapa Brunet, T; Delibes de Catro, G; Moure Romanillo,JA; Querol, MA; Santonja Gomez,M. Editorial Najera, Madrid.

PANOFSKY,E (1995): El significado en las artes visuales. Alianza Editorial.

RADCLIFFE-BROWN, A.R. (1986), Estructura u función en la sociedad primitiva. Agostini, Barcelona.

RIOPELLE, L. (1993): Cuidados de enfermería. Un proceso centrado en las necesidades de la persona. Editorial Interamericana. Mc. Graw-Hill.

SALMERÓN, J: Arte rupestre.

http://ayuntamiento.cieza.net/culturaypatrimonio/art erupestre/arte.html.

SILES GONZÁLEZ, J. 1999: Historia de la Enfermería volumen I y II. Consejo de enfermería de la Comunidad Valenciana.

SUREDA, J. (1985): Tomo I, Las primeras civilizaciones.en: Historia universal del arte. Dirigida por Jose Milicua. Editorial Planeta S:A: Barcelona.

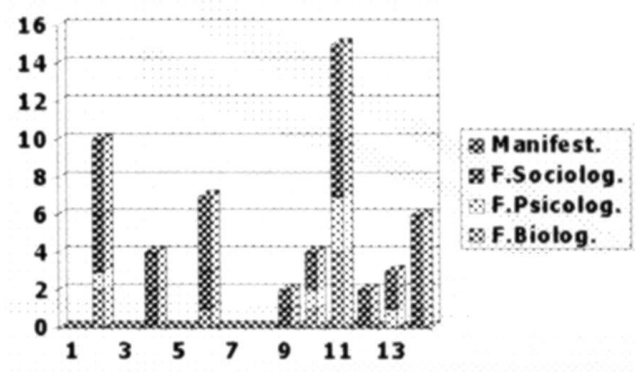

\section{Gráfico I}

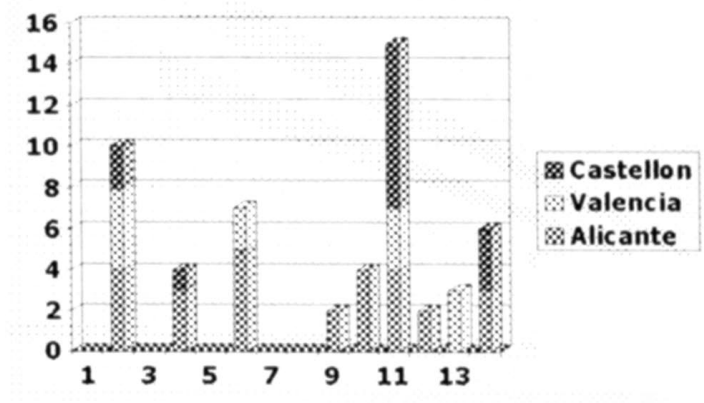

Gráfico 2 